$\mathrm{J}$ o u r n a l of

Mathematics

and Applications

JMA No 37, pp 111-118 (2014)

\title{
Certain subordination results on the convolution of analytic functions
}

\author{
Poonam Sharma, Rajesh K. Maurya
}

\begin{abstract}
In this paper, certain subordination results on the convolution of finite number of analytic functions are derived. Our results include a sufficiency condition for convexity of the convolution of analytic functions $f_{i}$ satisfying $f_{i}^{\prime} \in \mathcal{P}\left(\alpha_{i}\right)\left(\alpha_{i} \leq 1, i=1,2, \ldots, n\right)$.
\end{abstract}

AMS Subject Classification: $30 C 45$

Keywords and Phrases: Analytic function, Convolution (Hadamard product), Subordination, Convex functions, Zeta function

\section{Introduction}

Let $\mathcal{H}(\mathbb{U})$ denote a class of all analytic functions defined in the open unit disk $\mathbb{U}=$ $\{z \in \mathbb{C}:|z|<1\}$. For $a \in \mathbb{C}, j \in \mathbb{N}$, let

$$
\mathcal{H}[a, j]=\left\{f \in \mathcal{H}(\mathbb{U}): f(z)=a+a_{j} z^{j}+a_{j+1} z^{j+1}+\ldots\right\} .
$$

We denote the special class of $\mathcal{H}[0,1]$ by $\mathcal{A}$ whose members are of the form:

$$
f(z)=z+\sum_{k=2}^{\infty} a_{k} z^{k}, z \in \mathbb{U} .
$$

Let $\mathcal{K}$ denote a subclass of $\mathcal{A}$ whose members are convex (univalent) in $\mathbb{U}$ and satisfying

$$
\Re\left(1+\frac{z f^{\prime \prime}(z)}{f^{\prime}(z)}\right)>0, z \in \mathbb{U} .
$$

For two functions $p, q \in \mathcal{H}(\mathbb{U})$, we say $p$ is subordinate to $q$, or $q$ is superordinate to $p$ in $\mathbb{U}$ and write $p(z) \prec q(z), z \in \mathbb{U}$, if there exists a Schwarz function $\omega$, analytic in $\mathbb{U}$ with $\omega(0)=0$, and $|\omega(z)|<1, z \in \mathbb{U}$ such that $p(z)=q(\omega(z)), z \in \mathbb{U}$. Furthermore, if the function $q$ is univalent in $\mathbb{U}$, then we have following equivalence:

$$
p(z) \prec q(z) \Leftrightarrow p(0)=q(0) \text { and } p(\mathbb{U}) \subset q(\mathbb{U}) .
$$

COPYRIGHT (c) by Publishing Department Rzeszów University of Technology P.O. Box 85, 35-959 Rzeszów, Poland 
Let $\mathcal{P}_{\alpha}$ denote a class of functions $p \in \mathcal{H}(\mathbb{U})$ satisfying $p(0)=1$ and

$$
p(z) \prec q_{\alpha}(z):=\frac{1+(1-2 \alpha) z}{1-z}, \alpha \leq 1, z \in \mathbb{U} .
$$

Convolution (or Hadamard product) * of the functions $g_{1}(z)$ and $g_{2}(z)$ of the form:

$$
g_{1}(z)=\sum_{k=0}^{\infty} a_{k} z^{k} \text { and } g_{2}(z)=\sum_{k=0}^{\infty} b_{k} z^{k}
$$

is defined by

$$
g_{1}(z) * g_{2}(z)=\left(g_{1} * g_{2}\right)(z)=\sum_{k=0}^{\infty} a_{k} b_{k} z^{k}=\left(g_{2} * g_{1}\right)(z) .
$$

In 1973, Rusheweyh and Sheil-Small [3] proved the Pòlya-Schoenberg conjecture which shows that the convolution of two convex functions is again a convex function. Due to this convexity preserving property, attempts are made to involve and study convolutions in the Geometric Function Theory.

In this paper, we derive certain subordination results on the convolution of any finite number of analytic functions. Mainly, by applying the subordination principle, a sufficiency condition for convexity of $\phi(z):=\left(f_{1} * f_{2} * \ldots * f_{n}\right)(z)$ which is a convolution of analytic functions $f_{i} \in \mathcal{A}(i=1,2, \ldots, n)$ such that $f_{i}^{\prime} \in \mathcal{P}\left(\alpha_{i}\right)$ $\left(\alpha_{i} \leq 1, i=1,2, \ldots, n\right)$ is derived.

In order to obtain our results, we use following theorem of Rusheweyh and Stankiewicz [4]:

Theorem 1 Let $F, G \in \mathcal{H}(\mathbb{U})$ be any convex univalent functions in $\mathbb{U}$. If $f \prec F$ and $g \prec G$, then

$$
f * g \prec F * G \text { in } \mathbb{U} .
$$

Also, we use a result of Stankiewicz and Stankiewicz [6] which is as follows:

Theorem 2 If $\alpha \leq 1$ and $\beta \leq 1$, then

$$
P_{\alpha} * P_{\beta}=P_{\delta}
$$

where $\delta=1-2(1-\alpha)(1-\beta)$.

\section{Main Results}

We may easily generalize Theorem 2 for the classes $\mathcal{P}\left(\alpha_{i}\right)(i=1,2, \ldots, n)$ and get the following lemma:

Lemma 1 If $\alpha_{i} \leq 1(i=1,2, \ldots, n)$, then

$$
P_{\alpha_{1}} * P_{\alpha_{2}} * \ldots * P_{\alpha_{n}}=P_{\delta}
$$

where

$$
\delta=1-2^{n-1}\left(1-\alpha_{1}\right)\left(1-\alpha_{2}\right) \ldots\left(1-\alpha_{n}\right) .
$$


Theorem 3 Let for each $i=1,2, \ldots, n, f_{i} \in \mathcal{A}$ and $\alpha_{i} \leq 1$. If $f_{i}^{\prime} \in \mathcal{P}\left(\alpha_{i}\right)$ for each $i=1,2, \ldots, n$, and

$$
\phi(z)=\left(f_{1} * f_{2} * \ldots * f_{n}\right)(z)
$$

then

$$
\phi^{\prime}(z) \prec h(z), z \in \mathbb{U}
$$

where

$$
h(z)=1+2^{n}\left(1-\alpha_{1}\right)\left(1-\alpha_{2}\right) \ldots\left(1-\alpha_{n}\right)\left[\frac{z}{2^{n-1}}+\frac{z^{2}}{3^{n-1}}+\ldots\right]
$$

is convex univalent in $\mathbb{U}$.

Proof. Let $f_{i}^{\prime} \in \mathcal{P}\left(\alpha_{i}\right)$ for each $i=1,2, \ldots, n$. Then, in view of (1.3), we have for $\alpha_{i} \leq 1, i=1,2, \ldots, n, z \in \mathbb{U}$,

$$
f_{i}^{\prime}(z) \prec q_{\alpha_{i}}(z):=\frac{1+\left(1-2 \alpha_{i}\right) z}{1-z}=1+2\left(1-\alpha_{i}\right) \sum_{k=1}^{\infty} z^{k}
$$

where the superordinate functions $q_{\alpha_{i}}(z)$ for each $i=1,2, \ldots, n$ map the disk $\mathbb{U}$ onto convex univalent regions in the positive half plane. By Theorem 1, we get that

$$
f_{1}^{\prime}(z) * f_{2}^{\prime}(z) * \ldots * f_{n}^{\prime}(z) \prec q_{\alpha_{1}}(z) * q_{\alpha_{2}}(z) * \ldots * q_{\alpha_{n}}(z), z \in \mathbb{U}
$$

where

$$
\begin{aligned}
q_{\alpha_{1}}(z) * q_{\alpha_{2}}(z) * \ldots * q_{\alpha_{n}}(z) & =1+2^{n}\left(1-\alpha_{1}\right)\left(1-\alpha_{2}\right) \ldots\left(1-\alpha_{n}\right) \sum_{k=1}^{\infty} z^{k} \\
& =: \frac{1+(1-2 \delta) z}{1-z}, z \in \mathbb{U}
\end{aligned}
$$

is convex univalent in $\mathbb{U}$ and in view of Lemma $1, \delta$ is given by (2.1).

We know that the function

$$
h_{1}(z)=-\frac{2}{z}[z+\ln (1-z)]=\sum_{k=1}^{\infty} \frac{2}{k+1} z^{k}, z \in \mathbb{U}
$$

belongs to the class $\mathcal{K}$ and for $f \in \mathcal{A}$

$$
\left(f * h_{1}\right)(z)=\frac{2}{z} \int_{0}^{z} f(t) \mathrm{d} t .
$$

Therefore, the function

$$
h_{2}(z)=1+h_{1}(z), z \in \mathbb{U}
$$

being a translation of $h_{1}(z)$, is convex univalent in $\mathbb{U}$ and for $p \in \mathcal{H}[1,1]$

$$
\left(p * h_{2}\right)(z)=-1+\frac{2}{z} \int_{0}^{z} p(t) \mathrm{d} t .
$$


On applying, Theorem 1 , to the subordination (2.4) sequentially, $n-1$ times with the usual subordination: $h_{2}(z) \prec h_{2}(z), z \in \mathbb{U}$, we get

$$
f_{1}^{\prime} * f_{2}^{\prime} * \ldots * f_{n}^{\prime} * \underbrace{h_{2} * h_{2} * \ldots * h_{2}}_{n-1 \text { times }} \prec q_{\alpha_{1}} * q_{\alpha_{2}} * \ldots * q_{\alpha_{n}} * \underbrace{h_{2} * h_{2} * \ldots * h_{2}}_{n-1 \text { times }}
$$

in $\mathbb{U}$, which can also be written as

$$
\left(f_{1}^{\prime} * h_{2}\right) *\left(f_{2}^{\prime} * h_{2}\right) * \ldots *\left(f_{n-1}^{\prime} * h_{2}\right) * f_{n}^{\prime} \prec\left(q_{\alpha_{1}} * h_{2}\right) *\left(q_{\alpha_{2}} * h_{2}\right) * \ldots *\left(q_{\alpha_{n-1}} * h_{2}\right) * q_{\alpha_{n}} .
$$

On suitably choosing series expansions of $f_{i}^{\prime}$ 's and $q_{\alpha_{i}}$ 's, in view of (2.5), we observe that the subordination (2.6) reduces to

$$
\begin{aligned}
& \frac{f_{1}(z)}{z} * \frac{f_{2}(z)}{z} * \ldots * \frac{f_{n-1}(z)}{z} * f_{n}^{\prime}(z) \\
\prec & \frac{1}{z} \int_{0}^{z} q_{\alpha_{1}}(t) \mathrm{d} t * \frac{1}{z} \int_{0}^{z} q_{\alpha_{2}}(t) \mathrm{d} t * \ldots * \frac{1}{z} \int_{0}^{z} q_{\alpha_{n-1}}(t) \mathrm{d} t * q_{\alpha_{n}}(z) \\
= & h(z), z \in \mathbb{U}
\end{aligned}
$$

where $h(z)$ is convex univalent in $\mathbb{U}$ and is of the form (2.2). The left hand side (2.7) of above subordination is

$$
\left(f_{1} * f_{2} * \ldots * f_{n}\right)^{\prime}(z)=\phi^{\prime}(z) .
$$

This proves Theorem 3.

As the function $h(z)$ given by $(2.2)$ is convex univalent with real coefficients, we may easily get following result from Theorem 3 :

Corollary 1 Let for each $i=1,2, \ldots, n, f_{i} \in \mathcal{A}$ and $\alpha_{i} \leq 1$. If $f_{i}^{\prime} \in \mathcal{P}\left(\alpha_{i}\right)$ for each $i=1,2, \ldots, n$, and

$$
\phi(z)=\left(f_{1} * f_{2} * \ldots * f_{n}\right)(z)
$$

then

$$
h(-1) \leq \operatorname{Re}\left\{\phi^{\prime}(z)\right\} \leq h(1), z \in \mathbb{U}
$$

where $h(z)$ is given by (2.2).

In terms of Zeta function [[7], Ex.5, p.201], we may also find following result from Theorem 3:

Corollary 2 Let for each $i=1,2, \ldots, n, f_{i} \in \mathcal{A}$ and $\alpha_{i} \leq 1$. If $f_{i}^{\prime} \in \mathcal{P}\left(\alpha_{i}\right)$ for each $i=1,2, \ldots, n$, and

$$
\phi(z)=\left(f_{1} * f_{2} * \ldots * f_{n}\right)(z)
$$

then for $n>2$,

$$
\operatorname{Re}\left\{\phi^{\prime}(z)\right\} \geq 1+2^{n}\left(1-\alpha_{1}\right)\left(1-\alpha_{2}\right) \ldots\left(1-\alpha_{n}\right)\left[\left(1-2^{2-n}\right) \zeta(n-1)-1\right]
$$


and

$$
\operatorname{Re}\left\{\phi^{\prime}(z)\right\} \leq 1+2^{n}\left(1-\alpha_{1}\right)\left(1-\alpha_{2}\right) \ldots\left(1-\alpha_{n}\right)[\zeta(n-1)-1], z \in \mathbb{U}
$$

where $\zeta$ is well known Zeta Function. [5]:

Taking $n=3$ and writing $\zeta(2)=\frac{\pi^{2}}{6}$, Corollary 2 provides following result of Sokół

Corollary 3 Let for each $i=1,2,3, f_{i} \in \mathcal{A}$ and $\alpha_{i} \leq 1$. If $f_{i}^{\prime} \in \mathcal{P}\left(\alpha_{i}\right)$ for each $i=1,2,3$, and

$$
\phi(z)=\left(f_{1} * f_{2} * f_{3}\right)(z)
$$

then

$$
\operatorname{Re}\left\{\phi^{\prime}(z)\right\} \geq 1+8\left(1-\alpha_{1}\right)\left(1-\alpha_{2}\right)\left(1-\alpha_{3}\right)\left[\frac{\pi^{2}}{12}-1\right], z \in \mathbb{U}
$$

and

$$
\operatorname{Re}\left\{\phi^{\prime}(z)\right\} \leq 1+8\left(1-\alpha_{1}\right)\left(1-\alpha_{2}\right)\left(1-\alpha_{3}\right)\left[\frac{\pi^{2}}{6}-1\right], z \in \mathbb{U}
$$

To prove our next result, we prove first a lemma which is as follows:

Lemma 2 Let for each $i=1,2, \ldots, n, f_{i} \in \mathcal{A}$ and $\alpha_{i} \leq 1$. If $f_{i}^{\prime} \in \mathcal{P}\left(\alpha_{i}\right)$ for each $i=1,2, \ldots, n$, and

$$
\phi(z)=\left(f_{1} * f_{2} * \ldots * f_{n}\right)(z)
$$

then there exist some positive integers $\lambda_{1}, \lambda_{2}, \ldots, \lambda_{n-2}$ depending upon $n$ such that

$$
\begin{aligned}
& \phi^{\prime}(z)+\lambda_{1} z \phi^{\prime \prime}(z)+\lambda_{2} z^{2} \phi^{\prime \prime \prime}(z)+\ldots+\lambda_{n-2} z^{n-2} \phi^{(n-1)}(z)+z^{n-1} \phi^{(n)}(z) \\
= & \left(f_{1}^{\prime} * f_{2}^{\prime} * \ldots * f_{n}^{\prime}\right)(z) .
\end{aligned}
$$

Proof. Let $f_{i} \in \mathcal{A}$ be of the form

$$
f_{i}(z)=z+\sum_{k=2}^{\infty} a_{k}^{i} z^{k}, z \in \mathbb{U} .
$$

Then

$$
\left(f_{1}^{\prime} * f_{2}^{\prime} * \ldots * f_{n}^{\prime}\right)(z)=1+\sum_{k=2}^{\infty} k^{n} d_{k} z^{k-1}
$$

where

$$
d_{k}:=a_{k}^{1} a_{k}^{2} \ldots a_{k}^{n}, k \geq 2 .
$$

We note that for the functions $f_{i}(z), i=1,2, \ldots, n$, of the form $(2.8)$, the $r$-th $(r \in \mathbb{N})$ derivative of $\phi(z)$ is given by

$$
\phi^{(r)}(z)=\sum_{k=1}^{\infty} k(k-1) \ldots(k-r+1) d_{k} z^{k-r},
$$


where $d_{1}=1$ and for $k \geq 2, d_{k}$ is given by (2.10).

For some positive integers $\lambda_{1}, \lambda_{2}, \ldots, \lambda_{n-2}$ depending only upon $n$, we have for $k, n \in \mathbb{N}$, an identity :

$$
\begin{aligned}
k^{n} \equiv & k+\lambda_{1} k(k-1)+\lambda_{2} k(k-1)(k-2)+\ldots \\
& +\lambda_{n-2} k(k-1) \ldots(k-n+2)+k(k-1) \ldots(k-n+1) .
\end{aligned}
$$

For the positive integers $\lambda_{1}, \lambda_{2}, \ldots, \lambda_{n-2}$, appear in the identity (2.11) and with the use of this identity, we get

$$
\begin{aligned}
& \phi^{\prime}(z)+\lambda_{1} z \phi^{\prime \prime}(z)+\lambda_{2} z^{2} \phi^{\prime \prime \prime}(z)+\ldots+\lambda_{n-2} z^{n-2} \phi^{(n-1)}(z)+z^{n-1} \phi^{(n)}(z) \\
= & \sum_{k=1}^{\infty} k^{n} d_{k} z^{k-1}, \text { where } d_{1}=1 .
\end{aligned}
$$

This is the right hand side of (2.9). This proves Lemma 2.

Theorem 4 Let for each $i=1,2, \ldots, n, f_{i} \in \mathcal{A}$ and $\alpha_{i} \leq 1$. If $f_{i}^{\prime} \in \mathcal{P}\left(\alpha_{i}\right)$ for each $i=1,2, \ldots, n$, and

$$
\phi(z)=\left(f_{1} * f_{2} * \ldots * f_{n}\right)(z)
$$

then $\phi \in \mathcal{K}$ whenever for $n>2$,

$$
\left(1-\alpha_{1}\right)\left(1-\alpha_{2}\right) \ldots\left(1-\alpha_{n}\right) \leq \frac{3}{2^{n+2}\left[1-\left(1-2^{2-n}\right) \zeta(n-1)\right]}
$$

where $\zeta$ is well known Zeta Function.

Proof. Let $p(z)=\phi^{\prime}(z)$, then by Lemma 2 and by (2.4), we get

$$
\begin{aligned}
& \psi\left(p(z), z p^{\prime}(z), \ldots, z^{n-1} p^{(n-1)}(z)\right) \\
= & p(z)+\lambda_{1} z p^{\prime}(z)+\lambda_{2} z^{2} p^{\prime \prime}(z)+\ldots+\lambda_{n-2} z^{n-2} p^{(n-2)}(z)+z^{n-1} p^{(n-1)}(z) \\
= & \left(f_{1}^{\prime} * f_{2}^{\prime} * \ldots * f_{n}^{\prime}\right)(z) \prec \frac{1+(1-2 \delta) z}{1-z}, z \in \mathbb{U}
\end{aligned}
$$

where $\delta$ is given by (2.1). From Theorem 3, we have a possible solution of the above $n$-th order Euler-type differential subordination (2.13), as follows

$$
\phi^{\prime}(z)=p(z) \prec h(z), z \in \mathbb{U}
$$

where $h(z)$ is given by $(2.2)$.

The $r$-th $(r \in \mathbb{N})$ derivative of $h(z)$ is given by

$$
h^{(r)}(z)=2(1-\delta) \sum_{k=1}^{\infty} \frac{k(k-1) \ldots(k-r+1)}{(k+1)^{n-1}} z^{k-r} .
$$


For the positive integers $\lambda_{1}, \lambda_{2}, \ldots, \lambda_{n-2}$, appearing in the identity (2.11), we observe that

$$
\begin{aligned}
& \psi\left(h(z), z h^{\prime}(z), \ldots, z^{n-1} h^{(n-1)}(z)\right) \\
= & h(z)+\lambda_{1} z h^{\prime}(z)+\lambda_{2} z^{2} h^{\prime \prime}(z)+\ldots+\lambda_{n-2} z^{n-2} h^{(n-2)}(z)+z^{n-1} h^{(n-1)}(z) \\
= & 1+2(1-\delta) \sum_{k=1}^{\infty} z^{k}=\frac{1+(1-2 \delta) z}{1-z}, z \in \mathbb{U}
\end{aligned}
$$

where $\delta$ is given by (2.1). This verifies the admissiblity condition for $p(z)$ in $(2.14)$ to be a solution of the subordination (2.13).

Now, the function $\phi \in \mathcal{K}$ if

$$
1+\frac{z \phi^{\prime \prime}(z)}{\phi^{\prime}(z)} \prec \frac{1+z}{1-z}, z \in \mathbb{U}
$$

or

$$
1+\frac{z p^{\prime}(z)}{p(z)} \prec \frac{1+z}{1-z}, z \in \mathbb{U} .
$$

By [[1], Theorem 2.6b, p.60] the condition (2.15) implies

$$
p(z) \prec \frac{1}{(1-z)^{2}}, z \in \mathbb{U} .
$$

Thus, in view of (2.14), the function $\phi \in \mathcal{K}$ if

$$
h(z) \prec \frac{1}{(1-z)^{2}}, z \in \mathbb{U}
$$

that is if

$$
\min _{z \in \mathbb{U}} \Re\{h(z)\}=h(-1) \geq \frac{1}{4}
$$

which is the given condition (2.12) if we write the expression of $h(-1)$ (as it is written in Corollary 2) in terms of Zeta Function [[7], Ex.5, p.201]. This proves the result of Theorem 4.

Taking $n=3$ in Theorem 4 and on writing $\zeta(2)=\frac{\pi^{2}}{6}$, we get following result.

Corollary 4 Let for each $i=1,2,3, f_{i} \in \mathcal{A}$ and $\alpha_{i} \leq 1$. If $f_{i}^{\prime} \in \mathcal{P}\left(\alpha_{i}\right)$ for each $i=1,2,3$, then $\left(f_{1} * f_{2} * f_{3}\right)(z) \in \mathcal{K}$ whenever

$$
\left(1-\alpha_{1}\right)\left(1-\alpha_{2}\right)\left(1-\alpha_{3}\right) \leq \frac{9}{8\left(12-\pi^{2}\right)} \approx 0.53 .
$$

Remark 1 We remark that Corollary 4 improves the result of Sokót obtained in [[5], Theorem 2, 124].

\section{Acknowledgements.}

This work has been supported by J.R.F of C.S.I.R. (New Delhi, India) awarded to the second author

Authors are thankful to the referee for his/her useful comments. 


\section{References}

[1] S. S. Miller, P. T. Mocanu, Differential Subordinations, Marcel Dekker, Inc., 2000.

[2] St. Rusheweyh, Convolution in Geometric Function Theory, Les Presses de l'Univ. de Montreal, 1982.

[3] St. Ruscheweyh, T. Sheil-Small, Hadamard product of schlicht functions and the Poyla-Schoenberg conjecture, Comm. Math. Helv., 48 (1973), 119-135.

[4] St. Ruscheweyh, J. Stankiewicz, Subordination under convex univalent function, Bull. Pol. Acad. Sci. Math., 33 (1985), 499-502.

[5] J. Sokół, The convexity of Hadamard product of three functions, J. Math. Appl., 29 (2007), 121-125.

[6] J. Stankiewicz, Z. Stankiewicz, Some Applications of the Hadamard Convolution in the Theory of Functions, Ann. Univ. Mariae Curie-Sklodowska, 40 (1986), 251265.

[7] E. M. Stein and R. Shakarchi, Complex Analysis, Princeton University Press, New Jersey, USA

DOI: $10.7862 / \mathrm{rf} .2014 .10$

\section{Poonam Sharma}

email: sharma_poonam@lkouniv.ac.in

Rajesh K. Maurya

email: rajeshkrmaurya@gmail.com

Department of Mathematics

University of Lucknow, Lucknow 226007

Received 3.10.2013, Accepted 30.11.2013 